\title{
PREFACE (Vol 38, Issue1)
}

\author{
Nadia Magnenat-Thalmann ${ }^{1}$
}

Published online: 13 January 2022

(c) The Author(s), under exclusive licence to Springer-Verlag GmbH Germany, part of Springer Nature 2022

This first issue of the year 2022 contains 20 regular papers:

1. Gadipudi Amaranageswarao et al., "Joint restoration convolutional neural network for low-quality image super resolution"

2. Yuanfeng Zhou et al., "Probability driven approach for point cloud registration of indoor scene"

3. Yaman Dua et al., "Compression of multi-temporal hyperspectral images based on RLS filter"

4. Zhibo Rao et al., "Patch attention network with generative adversarial model for semi-supervised binocular disparity prediction"

5. Ping Wang et al., "Solving the PnL problem using the hidden variable method: an accurate and efficient solution"

6. Wajdi Elhamzi et al., "Brain tumor classification based on hybrid approach"

7. Xuemei Li et al., "Single-image super-resolution based on local biquadratic spline with edge constraints and adaptive optimization in transform domain"

8. Feng Liu et al., "Retinal vessel segmentation using simple SPCNN model and line connector"

9. Alireza Balavand et al., "A new feature clustering method based on crocodiles hunting strategy optimization algorithm for classification of MRI images"

10. Sudipta Mukhopadhyay et al., "A comparative study of single image fog removal methods"

11. Wen Wang et al., "An adaptive and rapid 3D Delaunay triangulation for randomly distributed point cloud data"

12. S. Berlin et al., "Spiking neural network based on joint entropy of optical flow features for human action recog- nition"

13. Abdenour Amamra et al., "Raw GIS to 3D road modeling for real-time traffic simulation"

14. Walid Barhoumi et al., "Adaptive feature selection in PET scans based on shared information and multi-label learning"

15. Wei Jiang et al., "Modality-transfer generative adversarial network and dual-level unified latent representation for visible thermal Person re-identification"

16. Anil Parihar et al., "A comprehensive survey on video frame interpolation techniques"

17. K. Santosh et al., "Weber local descriptor for image analysis and recognition: a survey"

18. Chengzhi Lyu et al., "Attention to fine-grained information: hierarchical multi-scale network for retinal vessel segmentation"

19. Changyan Xiao et al., "A corner-clustering method for detection of slab management numbers sprayed on steel slabs"

20. Monan Wang et al., "An improved simplified PCNN model for salient region detection"

Nadia Magnenat Thalmann.

Editor-in-Chief, the Visual Computer.

Publisher's Note Springer Nature remains neutral with regard to jurisdictional claims in published maps and institutional affiliations.
Nadia Magnenat-Thalmann

thalmann@miralab.ch

1 MIRALab-CUI, University of Geneva, Battelle, Building A, 7, Route de Drize, 1227 Carouge, Geneva, Switzerland 